\title{
GRMIN: A Heuristic Simplification Algorithm for Generalized Reed-Muller Expressions
}

\author{
Debatosh Debnath and Tsutomu Sasao \\ Department of Computer Science and Electronics \\ Kyushu Institute of Technology \\ Iizuka 820, Japan
}

\begin{abstract}
A generalized Reed-Muller expression (GRM) is a type of AND-EXOR expressions. In a GRM, each variable may appear both complemented and uncomplemented. Networks realized using GRMs are easily tested. This paper presents GRMIN, a heuristic simplification algorithm for GRMs of multiple-output functions. GRMIN uses eight rules. As the primary objective, it reduces the number of products, and as the secondary objective, it reduces the number of literals. Experimental results show that, in most cases, GRMs require fewer products than conventional sum-of-products expressions (SOPs). GRMIN outperforms existing algorithms.
\end{abstract}

\section{INTRODUCTION}

Logic networks are usually designed by using AND and OR gates. However, networks using exclusive-OR (EXOR) gates have some advantages over conventional AND-OR networks. First, such networks often require fewer gates and interconnections than those designed using AND and OR gates [13]. Examples of such networks include, arithmetic, telecommunication, and error correcting circuits. Second, they can be made easily testable.

Various classes exist in AND-EXOR expressions $[6,12]$. Among them, positive polarity Reed-Muller expressions (PPRMs) are well known: a PPRM, an EXOR sum-ofproducts with positive literals, uniquely represents an arbitrary logic function. Networks based on PPRMs are easily testable [10], but they often require more products than ones based on other expressions. Generalized Reed-Muller expressions (GRMs) [4] are generalization of PPRMs. They never require more products than PPRMs, and often require many fewer products. GRMs were studied many years ago [2], but no practical applications have been reported. Recently, easily testable realizations for GRMs have been developed [14]. Because GRMs often require many fewer products than PPRMs and have very good testability, the GRM based design has practical importance. An exact minimization algorithm for GRMs is available [15], but for the functions with more than six variables it is very time and memory consuming. For heuristic simplification of GRMs, only a few algorithms [3, 9] are available. In this paper, we present GRMIN, a heuristic simplification algorithm for GRMs of multiple-output function. Experimental results show that GRMIN outperforms existing algorithms.

\section{Definitions and Basic Properties}

\subsection{Positional Cube Notation}

A positional cube [7] is convenient for manipulation of logic expressions by computers. In positional cube notation, an uncomplemented literal such as $x_{i}$ is represented by 01 , a complemented literal $\bar{x}_{i}$ is represented by 10 , and a don't care (missing variable in a product) is represented by 11 . 00 represents no value of the variable, and any cube containing a 00 for any variable position denotes a null cube.

For $m$-output functions $\left(f_{0}, f_{1}, \ldots, f_{m-1}\right)(m \geq 1)$, an $m$-bit tag field is concatenated to the cube to denote the output. If the $i$ th bit of the tag field of a cube is 1 , the $i$ th output is occupied by the cube. In this case, we treat the outputs as an $m$-valued variable.

In the positional cube notation, each variable constitutes a part. The $m$-bit tag constitutes the output part. Thus, in the representation of an $n$-variable function there are $n+1$ parts.

Definition 2.1 A list of cubes is called the OR array if it represents the $O R$ of cubes, and called the $\mathbf{E X O R}$ array if it represents the EXOR of cubes.

Example 2.1 The three-variable three-output function $f_{0}=x_{1} \bar{x}_{3} \oplus x_{2} x_{3}, f_{1}=x_{2} x_{3}$, and $f_{2}=x_{1} \bar{x}_{3}$ is represented by the four part EXOR array:

$$
\begin{aligned}
& \frac{x_{1} \quad x_{2} \quad x_{3} \quad f_{0} f_{1} f_{2}}{01-11-10-101} \\
& 11-01-01-110 . \text { (End of Example) }
\end{aligned}
$$

From now on, unless otherwise specified, a list of cube represents an EXOR array.

Definition 2.2 The c-distance between two cubes is the number of parts in which they differ.

Example 2.2 The c-distance between the two cubes in Example 2.1 is four.
(End of Example) 


\subsection{Logical Expression}

An alternate representation of a logic function is a logical expression. In this representation, position of 1 's in a part of the positional cube notation of a cube are shown as the exponent of the corresponding variable. For example, $x^{\{0\}}, x^{\{1\}}$, and $x^{\{0,1\}}$ is used to represent 10,01 , and 11, respectively. In Section 3.3, this representation is used to show the simplification rules for GRMs. From now on, we will consider the binary outputs as a single multiple-valued output and represent by $z$. All the inputs are two-valued.

Example 2.3 The three-variable multiple-output function shown in Example 2.1 can be represented by $x_{1}^{\{1\}} x_{2}^{\{01\}} x_{3}^{\{0\}} z^{\{02\}} \oplus x_{1}^{\{01\}} x_{2}^{\{1\}} x_{3}^{\{1\}} z^{\{01\}}$. Because it is an EXOR array, the $\oplus$ operator is used. (End of Example)

\subsection{PPRM, FPRM and GRM}

In this part, we will define three classes of AND-EXOR expressions. The following lemma is the basis of the EXOR-based expansion [12]:

Lemma 2.1 An arbitrary logic function $f\left(x_{1}, x_{2}, \ldots, x_{n}\right)$ can be expanded as

$$
\begin{aligned}
& f=\bar{x}_{1} f_{0} \oplus x_{1} f_{1}, \\
& f=f_{0} \oplus x_{1} f_{2}, \\
& f=f_{1} \oplus \bar{x}_{1} f_{2},
\end{aligned}
$$

where $f_{0}=f\left(0, x_{2}, \ldots, x_{n}\right), f_{1}=f\left(1, x_{2}, \ldots, x_{n}\right)$, and $f_{2}=f_{0} \oplus f_{1}$.

(2.1), (2.2), and (2.3) are called the Shannon expansion, the positive Davio expansion, and the negative Davio expansion, respectively. If we use (2.2) recursively to a function $f$, then we have the following:

Lemma 2.2 An arbitrary n-variable function $f\left(x_{1}, x_{2}, \ldots, x_{n}\right)$ can be represented as

$$
\begin{aligned}
f= & a_{0} \oplus a_{1} x_{1} \oplus a_{2} x_{2} \oplus \cdots \oplus a_{n} x_{n} \\
& \oplus a_{12} x_{1} x_{2} \oplus a_{13} x_{1} x_{3} \oplus \cdots \oplus a_{n-1} x_{n-1} x_{n} \oplus \\
& \cdots \cdots \cdots \cdots \cdots \cdots \cdots \cdots \cdots \cdots \\
& \oplus a_{12 \cdots n} x_{1} x_{2} x_{3} \cdots x_{n}
\end{aligned}
$$

where a's are either 0 or 1 .

(2.4) is called a positive polarity Reed-Muller expression (PPRM). For a given function $f$, the coefficients $a_{0}, a_{1}, a_{2}, \ldots, a_{12 \cdots n}$ are uniquely determined. Thus, the PPRM is a canonical representation. The number of products in (2.4) is at most $2^{n}$, and all the literals are positive (uncomplemented).

In $(2.4)$, for each variable $x_{i}(i=1,2, \ldots, n)$, if we use either a positive literal $\left(x_{i}\right)$ throughout or a negative literal $\left(\bar{x}_{i}\right)$ throughout, then we have a fixed polarity Reed-Muller expression (FPRM). For each variable $x_{i}$, there are two ways of choosing the polarities: positive $\left(x_{i}\right)$ or negative $\left(\bar{x}_{i}\right)$. Thus, $2^{n}$ different set of polarities exist for an $n$-variable function. For a given function and a given set of polarities, a unique set of coefficients $\left(a_{0}, a_{1}, \ldots, a_{12 \cdots n}\right)$ exists. Thus, an FPRM is a canonical representation.

In (2.4), if we can freely choose the polarity for each literal, then we have a generalized Reed-Muller expression (GRM). Unlike FPRMs, both $x_{i}$ and $\bar{x}_{i}$ can appear in a GRM. Some authors use GRMs to represent another class of expressions [6], thus the terminology is not unified. There are $n 2^{n-1}$ literals in (2.4), so $2^{n 2^{n-1}}$ different set of polarities exist for an $n$-variable function. For a given set of polarities, a unique set of coefficients $\left(a_{0}, a_{1}, \ldots, a_{12 \cdots n}\right)$ exists. Thus, a GRM is a canonical representation for a logic function.

A GRM for a multiple-output function is defined as follows:

Definition 2.3 An array represents a multiple-output GRM, if for each output, the corresponding cubes represent a GRM.

Example 2.4 Consider an array representing a threevariable two-output function:

$$
\begin{array}{lr}
x_{1} x_{2} & x_{3} \quad f_{0} f_{1} \\
\hline 10-10-10-10 \\
10-01-01-01 \\
01-01-11-11 .
\end{array}
$$

Both $f_{0}=\bar{x}_{1} \bar{x}_{2} \bar{x}_{3} \oplus x_{1} x_{2}$, and $f_{1}=\bar{x}_{1} x_{2} x_{3} \oplus x_{1} x_{2}$ are GRMs. Thus, the array represents a multiple-output GRM.

(End of Example)

\subsection{PSDRM and ESOP}

Before studying the simplification method for GRMs, it is convenient to define other classes of expressions.

Suppose that we are given a three-variable function $f\left(x_{1}, x_{2}, x_{3}\right)$. When we expand $f$ using the positive Davio expansion with respect to $x_{1}$, we have

$$
f=f_{0} \oplus x_{1} f_{2} .
$$

Next, when we expand $f_{0}$ and $f_{2}$ in a similar way with respect to $x_{2}$, we have

$$
f_{0}=f_{00} \oplus x_{2} f_{02}, f_{2}=f_{20} \oplus x_{2} f_{22} .
$$

Furthermore, when we use similar expansions with respect to $x_{3}$, we have

$$
\begin{aligned}
& f_{00}=f_{000} \oplus x_{3} f_{002}, f_{02}=f_{020} \oplus x_{3} f_{022}, \\
& f_{20}=f_{200} \oplus x_{3} f_{202}, \quad f_{22}=f_{220} \oplus x_{3} f_{222} .
\end{aligned}
$$

The expansion tree in Fig. 2.1 illustrates this process. A path from the root node to a terminal node represents a product of an expression, where a label of an edge shows the literal for the corresponding variable. For example, the path from the root node to $f_{000}$ represents the product $1 \cdot 1 \cdot 1 \cdot f_{000}=f_{000}$, and the path to $f_{222}$ represents $x_{1} x_{2} x_{3} f_{222}$. Thus, the tree in Fig. 2.1 shows the PPRM:

$$
\begin{aligned}
f= & f_{000} \oplus x_{3} f_{002} \oplus x_{2} f_{020} \oplus x_{2} x_{3} f_{022} \oplus x_{1} f_{200} \\
& \oplus x_{1} x_{3} f_{202} \oplus x_{1} x_{2} f_{220} \oplus x_{1} x_{2} x_{3} f_{222} .
\end{aligned}
$$




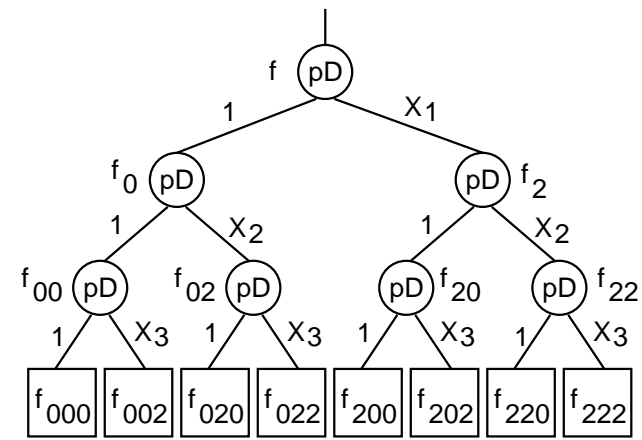

Fig. 2.1. Representation of a logic function using positive Davio expansions.

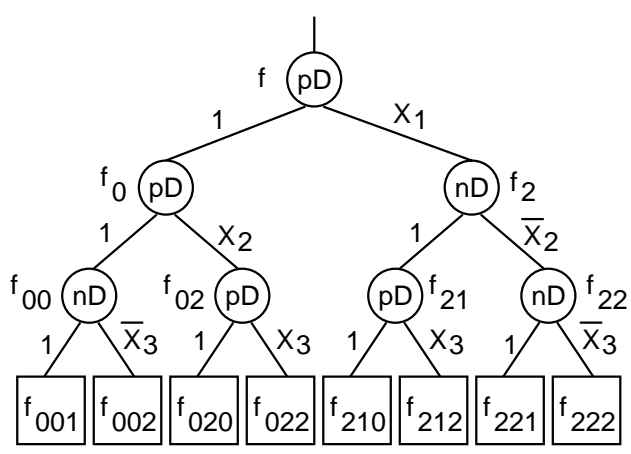

Fig. 2.2. Representation of a logic function using pseudo Reed-Muller expansions.

Each node has a label pD, which shows the positive Davio expansion. In Fig. 2.1, only the positive Davio expansions are used. However, if we use either the positive or the negative Davio expansion for each variable, then we have a more general tree. Such a tree represents an FPRM. If we use either the positive or the negative Davio expansion for each node, then we have a more general tree. Such a tree represents a pseudo Reed-Muller expression (PSDRM). For example, in Fig. 2.2, $f, f_{0}, f_{02}$, and $f_{21}$ use the positive Davio expansions, while $f_{2}, f_{00}$, and $f_{22}$ use the negative Davio expansions. Nodes with label $\mathrm{nD}$ denotes the negative Davio expansion. Note that the tree in Fig. 2.2 shows the PSDRM:

$$
\begin{aligned}
f= & 1 \cdot 1 \cdot 1 \cdot f_{001} \oplus 1 \cdot 1 \cdot \bar{x}_{3} f_{002} \oplus 1 \cdot x_{2} \cdot 1 \cdot f_{020} \\
& \oplus 1 \cdot x_{2} x_{3} f_{022} \oplus x_{1} \cdot 1 \cdot 1 \cdot f_{210} \oplus x_{1} \cdot 1 \cdot x_{3} f_{212} \\
& \oplus x_{1} \bar{x}_{2} \cdot 1 \cdot f_{221} \oplus x_{1} \bar{x}_{2} \bar{x}_{3} f_{222} .
\end{aligned}
$$

Arbitrary set of product terms combined by EXORs is called an Exclusive-or Sum-of-Products Expression (ESOP). The ESOP is the most general AND-EXOR expression. Arbitrary set of product terms combined by ORs is called a Sum-of-Products Expression (SOP).

\section{Example 2.5}

1. $x_{1} \oplus x_{2} \oplus x_{1} x_{2}$ is a PPRM (all literals are uncomplemented).
2. $x_{1} \oplus \bar{x}_{2} \oplus x_{1} \bar{x}_{2}$ is an FPRM, but not a PPRM $\left(x_{2}\right.$ have complemented literals).

3. $x_{2} \oplus x_{1} \bar{x}_{2}$ is a PSDRM, but not an FPRM ( $x_{2}$ have both complemented and uncomplemented literals).

4. $x_{1} \oplus x_{2} \oplus \bar{x}_{1} \bar{x}_{2}$ is a GRM, but not a PSDRM (it cannot be generated by an expansion tree for a PSDRM).

\subsection{Properties of GRMs}

In this part, we consider some properties of GRMs, useful for the simplification of expressions.

Definition 2.4 The variable set of a product $p$ is denoted by $V(p)=\left\{x_{i} \mid x_{i}\right.$ or $\bar{x}_{i}$ appears in $\left.p\right\}$. The variable set of a cube is the variable set of the product represented by the input parts of the cube.

Example 2.6 $V\left(x_{1} \bar{x}_{2} \bar{x}_{4}\right)=\left\{x_{1}, x_{2}, x_{4}\right\}$. The variable sets of the first and second cubes in Example 2.1 are $\left\{x_{1}, x_{3}\right\}$, and $\left\{x_{2}, x_{3}\right\}$, respectively. (End of Example)

From the definition of GRMs, we have the following lemma:

Lemma 2.3 An AND-EXOR expression (for singleoutput function) is a GRM, iff no two products have the same set of variables.

Example 2.7 Let $f=x_{4} \oplus x_{1} x_{2} x_{3} \oplus \bar{x}_{1} \bar{x}_{2} \bar{x}_{3}$. Then $V\left(x_{1} x_{2} x_{3}\right)=\left\{x_{1}, x_{2}, x_{3}\right\}$, and $V\left(\bar{x}_{1} \bar{x}_{2} \bar{x}_{3}\right)=\left\{x_{1}, x_{2}, x_{3}\right\}$. Thus, $f$ is not a GRM because two products have the same set of variables.

(End of Example)

In a GRM for multiple-output function, more than one cube may have the same set of variables.

Lemma 2.4 An array represents a multiple-output GRM, if for any output, no two cubes have the same set of variables.

(Proof) Let the array represent $m$ single output functions, $f_{i}(i=0, \ldots, m-1)$. If the $i$ th bit of the output part of a cube is $1, f_{i}$ contains the corresponding cube. From the hypothesis of the lemma, no two cubes of $f_{i}$ have the same set of variables. So, by Lemma $2.3, f_{i}$ represents a GRM of single output function. Thus, from the Definition 2.3, we have the lemma.

(Q.E.D.)

Corollary 2.1 In an array for a multiple-output function, if cubes having the same set of variables have nondisjoint output parts, then the array does not represent a GRM.

Example 2.8 Consider the array in Example 2.4. The first and the second cubes have the same set of variables, but their output parts are disjoint. Thus, the array represents a multiple-output GRM. 


\section{Simplification Algorithm}

\subsection{Outline of GRMIN}

GRMIN has the following features:

1. As an input, it accepts a PSDRM, or quickly generates good initial solutions from the given SOP.

2. It simplifies multiple-output functions.

3. It uses eight rules iteratively to reduce the number of products.

4. It modifies the cubes repeatedly by replacing a pair of cubes with another one, while keeping the array to represent a GRM.

5. When reduction of the number of products become impossible in the iterative improvement mode, it temporarily increases the number of products, and simplifies again. An increase in the number of products, increases the computation time, but often reduce the number of products in the final solution [1].

\subsection{Initial Solution}

As an initial solution, GRMIN accepts any GRMs. Alternatively, one can minimize two GRMs from two different initial solutions and take the one with fewer products.

PSDRMs: PPRMs, FPRMs and PSDRMs are special classes of GRMs, and any of them can be used as an initial solution for the GRM. We use a PSDRM as an initial solution, because minimal PSDRMs are easy to derive and usually require fewer products than PPRMs and FPRMs. We used the algorithm in [12] to obtain PSDRMs. For functions with up to 14-variables the algorithm quickly produce good initial solutions on an ordinary workstation.

Modified DSOPs: If each pair of products in an SOP are disjoint, then it is called a disjoint SOP (DSOP). In a DSOP, the OR operators can be replaced by the EXOR operators without changing the function represented by the expression. To obtain an initial solution, from the given SOP, first we obtain a DSOP [13]. Then, we use some heuristic to remove the cubes having the same set of variables for a output (Lemma 2.4).

\subsection{Simplification Rules}

Definition 3.1 $\cap$ and $\cup$ denote the intersection and union operation between two sets, respectively. Also '-, (overline) denotes the complement of a set. If $A$ and $B$ are sets, $A \oplus B=(A \cap \bar{B}) \cup(\bar{A} \cap B)$. The symbol $\oplus$ is also used to denote the EXOR of two logic functions. A set is null $(\phi)$ if it contains no element.

For simplification of GRMs, we use eight rules. Let $A, B, C, D \subseteq P$, where $P=\{0,1\}$. Then, the simplification rules for GRMs are as follows:

\section{X-MERGE}

$$
X^{A} \oplus X^{B} \Rightarrow X^{(A \oplus B)}
$$

2. RESHAPE

$$
\begin{gathered}
X^{A} Y^{B} \oplus X^{C} Y^{D} \Rightarrow X^{A} Y^{(B \cap \bar{D})} \oplus X^{(A \cup C)} Y^{D} \\
\text { if }(A \cap C=\phi, B \supset D)
\end{gathered}
$$

3. DUAL-COMPLEMENT

$$
\begin{gathered}
X^{A} Y^{B} \oplus X^{C} Y^{D} \Rightarrow X^{(\bar{A} \cap C)} Y^{B} \oplus X^{C} Y^{(B \cap \bar{D})} \\
\text { if }(A \subset C, B \supset D)
\end{gathered}
$$

4. X-EXPAND-1

$$
\begin{aligned}
X^{A} Y^{B} \oplus X^{C} Y^{D} & \Rightarrow X^{A} Y^{(B \cup D)} \oplus X^{(A \cup C)} Y^{D} \\
& \Rightarrow X^{(A \cup C)} Y^{B} \oplus X^{C} Y^{(B \cup D)} \\
\text { if }(A \cap C & =\phi, B \cap D=\phi)
\end{aligned}
$$

5. X-EXPAND-2

$$
\begin{gathered}
X^{A} Y^{B} \oplus X^{C} Y^{D} \Rightarrow X^{(A \cup C)} Y^{B} \oplus X^{C} Y^{(B \cap \bar{D})} \\
\text { if }(A \cap C=\phi, B \supset D)
\end{gathered}
$$

6. X-REDUCE-1

$$
\begin{gathered}
X^{A} Y^{B} \oplus X^{C} Y^{D} \Rightarrow X^{(A \cap \bar{C})} Y^{B} \oplus X^{C} Y^{(D \cap \bar{B})} \\
\text { if }(A \supset C, B \subset D)
\end{gathered}
$$

\section{X-REDUCE-2}

$$
\begin{gathered}
X^{A} Y^{B} \oplus X^{C} Y^{D} \Rightarrow X^{(A \cap \bar{C})} Y^{B} \oplus X^{C} Y^{(B \cap \bar{D})} \\
\text { if }(A \supset C, B \supset D)
\end{gathered}
$$

\section{COMPLEMENT}

$$
X^{A} \Rightarrow X^{\bar{A}} \oplus X^{P}
$$

The representation, analysis, and proof of these rules for multiple-valued input functions can be found in [13]. All the rules except for COMPLEMENT are also used in EXMIN2 [13] to simplify ESOPs.

\subsection{Examples of Simplification}

Example 3.1 Consider the following array:

$$
\begin{array}{cc}
x_{1} x_{2} \quad f_{0} f_{1} \\
\hline 10-11-01 \\
01-10-01 \\
11-10-10 .
\end{array}
$$

$X$-MERGE is inapplicable to this array, but RESHAPE is applicable to the first two cubes, and we have the following array:

$$
\begin{array}{cc}
x_{1} x_{2} & f_{0} f_{1} \\
\hline 10-01-01 \\
11-10-01 \\
11-10-10 .
\end{array}
$$

By merging the last two cubes, we have a GRM with two cubes:

$$
\begin{aligned}
& \frac{x_{1} x_{2} \quad f_{0} f_{1}}{10-01-01} \\
& 11-10-11 . \quad \text { (End of Example) }
\end{aligned}
$$

\subsection{Properties of the Simplification Rules ${ }^{1}$}

To simplify GRMs we use the eight rules of Section 3.3. Among them, X-MERGE is the only rule that reduces the number of cubes. When X-MERGE becomes inapplicable, other rules are used to modify the shape of the cubes so that X-MERGE becomes applicable. For a pair

\footnotetext{
${ }^{1}$ The proof of the lemmas can be found from the authors.
} 
of cubes, the rules (1-7) are applicable only when the cdistance between them is one or two. The COMPLEMENT operation is used to increase the number of products. During simplification, the array is kept to represent a GRM.

Lemma 3.1 In an array for a GRM, if the output parts of two cubes differ, then RESHAPE is inapplicable.

Lemma 3.2 For a pair of cubes in an array for GRM, if two input parts differ, then $X-E X P A N D-1$ is inapplicable.

Lemma 3.3 For a pair of cubes in an array for GRM, if they are disjoint in an input part, and differ in the output part, then $X-E X P A N D-2$ is inapplicable.

Lemma 3.4 In an array for GRM, if a pair of cubes differs in two input parts, then $X-R E D U C E-1$ is inapplicable.

Rules 2-8 of Section 3.3 may produce cubes with new variable sets and modified output parts. Therefore, before applying these rules, we check if the resultant array represents a GRM or not. If it is non-GRM, we discard the operation to keep the array to represent a GRM.

Example 3.2 Consider an array for a GRM:

$$
\begin{gathered}
x_{1} x_{2} \quad x_{3} \quad f_{0} f_{1} f_{2} \\
\hline 10-10-11-110 \\
10-01-01-110 \\
01-11-10-011 .
\end{gathered}
$$

By applying RESHAPE to the first two cubes, we have

$$
\begin{gathered}
x_{1} x_{2} x_{3} \quad f_{0} f_{1} f_{2} \\
\hline 10-10-10-110 \\
10-11-01-110 \\
01-11-10-011 .
\end{gathered}
$$

The last two cubes of this array have identical variable sets, i.e., $\left\{x_{1}, x_{3}\right\}$, and the outputs are non-disjoint. Thus, the array no longer represents a GRM, and we discard this operation and keep the array to represent a GRM.

(End of Example)

Lemma 3.5 In an array for a GRM, if a rule does not change the variable set of a cube, and produces outputs which are a subset of the original outputs, then the cube can be modified without checking the array.

Example 3.3 Consider an array of $k(k>3)$ cubes for a GRM. Let three of the cubes in the array be:

$$
\begin{gathered}
x_{1} x_{2} x_{3} \quad f_{0} f_{1} f_{2} \\
\hline 01-10-01-101 \\
01-10-11-001 \\
10-01-11-110 .
\end{gathered}
$$

By applying DUAL-COMPLEMENT to the first two cubes, we have

$$
\begin{gathered}
x_{1} x_{2} x_{3} \quad f_{0} f_{1} f_{2} \\
\hline 01-10-10-101 \\
01-10-11-100 \\
10-01-11-110
\end{gathered}
$$

In the first cube, the variable set remains the same and the outputs are the subset of the original outputs (equal here). Thus, we can modify the first cube without checking the array. Note that the polarity of the literal $x_{3}$ has been changed.

In the second cube, the variable set remains the same but the outputs are not a subset of the original outputs. So, we have to check if the resultant array represents a GRM or not. The second and third cube have the same set of variables and the outputs are non-disjoint. Thus, the array no longer represent a GRM, and we have to discard this operation to keep the array represent a GRM.

(End of Example)

Lemma 3.6 In an array for GRM, if a pair of cubes differs in two input parts, and DUAL-COMPLEMENT is applied, then the cubes can be modified without checking other cubes in the array.

\subsection{Algorithm}

For many functions, the order of the simplification rules influences the final solution. Currently, we are using the following heuristic algorithm.

\section{Algorithm 3.1 (GRMIN: Simplification of GRMs)}

1. Obtain an initial solution from the given SOP (Section 3.2).

2. For each pair of cubes, check if $X-M E R G E$ is applicable. If so, $X-M E R G E$ them. Continue this step while reductions of the number of cubes are possible.

3. For each pair of cubes, check if RESHAPE, DUALCOMPLEMENT, X-REDUCE-1, or X-REDUCE-2 are applicable. If so, apply that.

4. For each pair of cubes, check if $X-M E R G E$ is applicable. If so, $X$-MERGE them. Continue this step while reductions of the number of cubes are possible.

5. If the number of cubes is reduced in step 4, then go to step 3.

6. For each pair of cubes, check if X-EXPAND-1, XEXPAND-2, RESHAPE, or DUAL-COMPLEMENT are applicable. If so, apply that.

7. For each pair of cubes, check if $X-M E R G E$ is applicable. If so, $X$-MER GE them. Continue this step while reductions of the number of cubes are possible.

8. If the number of cubes is reduced in step 7 , then go to step 6.

9. If the number of cubes is reduced between steps 3-8, then go to step 3.

10. Temporarily increase the number of products by COMPLEMENT, and simplify using steps 2-9. Continue this step while reductions of the number of cubes are possible. 
TABLE 4.1

Comparison with Cannes [3]

\begin{tabular}{|l|r|c|c|c|c|}
\hline Data & In & Out & Cannes & GRMIN & Improvement \\
\hline 5xp1 & 7 & 10 & 60 & 35 & $42 \%$ \\
con1 & 7 & 2 & 12 & 9 & $25 \%$ \\
misex1 & 8 & 7 & 20 & 13 & $35 \%$ \\
rd53 & 5 & 3 & 20 & $20 *$ & $0 \%$ \\
rd73 & 7 & 3 & 63 & $63 *$ & $0 \%$ \\
sao2 & 10 & 4 & 52 & 28 & $46 \%$ \\
squar5 & 5 & 8 & 22 & 19 & $14 \%$ \\
sym9 & 9 & 1 & 131 & $126 *$ & $4 \%$ \\
xor5 & 5 & 1 & 5 & $5 *$ & $0 \%$ \\
\hline
\end{tabular}

* Exact minimum GRM.

11. For each pair of cubes, check if $X-R E D U C E-1$, $X-E X P A N D-2$ (when two input parts differ), $R E$ SHAPE, or DUAL-COMPLEMENT (when two input parts differ), are applicable. If so, apply that. Continue this step while reductions of the number of connections are possible.

12. Check that the simplified cubes represent a GRM, and verify that it is functionally equivalent to the given $S O P$.

In steps 2, 4, and 7, more than one merging passes are often required. In our implementation, we used additional fields for each cube. Using these fields, we can avoid many redundant computations. One of these fields stores when the cube was modified. For example, during the first merging pass in step 4 of Algorithm 3.1, it checks two cubes if at least one of them was modified in step 3 . As stated in Section 3.5, before applying a rule, the algorithm checks the array to determine if it represents a GRM. Another field is used to make this checking easier by storing variable set information.

\section{Experimental Results}

We coded GRMIN in C. As an initial solution, it accepts a PSDRM, or generates an initial GRM from the given SOP. Table 4.1 compares the number of products generated by GRMIN with that of Cannes [3], another heuristic simplification program for GRMs. It shows that GRMIN outperforms Cannes, and the improvement is up to $46 \%$. For rd53, rd73, sym9, and xor5, GRMIN produced exact minimum solutions (denoted by ${ }^{*}$ ). To show the minimality, we obtained lower bounds on the number of products in GRM [15].

Table 4.2 compares the number of products required to realize different arithmetic functions by various ANDEXOR expressions. In this experiment, PPRMs, FPRMs, and PSDRMs were minimized by a program in [12]; ESOPs were simplified by EXMIN2 [13]; and GRMs were simplified by GRMIN. For adr4, inc8, sym9, and wgt8, GRMIN produced exact minimum solutions. This table also shows that GRMs for arithmetic functions often require many fewer products than SOPs.
TABLE 4.2

Number OF PRODUCTS TO REALIZE ARITHMETIC FUNCTIONS

\begin{tabular}{|l|c|c|c|c|c|r|}
\hline Data & PPRM & FPRM & PSDRM & GRM & ESOP & SOP \\
\hline adr4 & 34 & 34 & 34 & $34 *$ & 31 & 75 \\
inc8 & 16 & 16 & 16 & $15 *$ & 15 & 37 \\
log8 & 253 & 193 & 163 & 105 & 96 & 123 \\
mlp4 & 97 & 97 & 90 & 71 & 61 & 121 \\
nrm4 & 216 & 185 & 150 & 96 & 69 & 120 \\
rdm8 & 56 & 56 & 46 & 31 & 31 & 76 \\
rot8 & 225 & 118 & 81 & 51 & 35 & 57 \\
sqr8 & 168 & 168 & 164 & 121 & 112 & 178 \\
sym9 & 210 & 173 & 127 & $126 *$ & 51 & 84 \\
wgt8 & 107 & 107 & 107 & $107 *$ & 58 & 255 \\
\hline
\end{tabular}

*Exact minimum GRM.

TABLE 4.3

Number OF PRODUCTS TO REALIZE RANDOMLY GENERATED FUNCTIONS

\begin{tabular}{|r|r|r|r|r|r|r|r|}
\hline$n$ & $|f|$ & PPRM & FPRM & PSDRM & GRM & ESOP & SOP \\
\hline 4 & 8 & 6 & 5 & 4 & $4^{*}$ & 3 & 4 \\
5 & 16 & 16 & 10 & 7 & $5 *$ & 5 & 6 \\
6 & 32 & 36 & 17 & 13 & $10 *$ & 10 & 13 \\
7 & 64 & 64 & 54 & 30 & 19 & 19 & 24 \\
8 & 128 & 122 & 101 & 56 & 36 & 36 & 46 \\
9 & 256 & 236 & 226 & 112 & 66 & 64 & 86 \\
10 & 512 & 528 & 459 & 235 & 142 & 142 & 167 \\
11 & 1024 & 1021 & 956 & 458 & 275 & 274 & 331 \\
12 & 2048 & 1996 & 1925 & 909 & 542 & 539 & 611 \\
13 & 4096 & 4136 & 3923 & 1813 & 1098 & 1045 & 1157 \\
14 & 8192 & 8210 & 7924 & 3617 & 2205 & 2150 & 2234 \\
\hline
\end{tabular}

*Exact minimum GRM.

Table 4.3 compares the number of products to realize randomly generated functions. For each value of $n$, an $n$ variable pseudo-random function with $2^{n-1}$ minterms was generated and minimized. Here $|f|$ denotes the number of true minterms of the function. For up to 6-variable functions, we verified that GRMIN produced exact minimum solutions by using an exact minimization program [15]. In this experiment, SOPs were simplified by MINI II [12], and other data were obtained by the same programs as mentioned above. This table shows that for randomly generated functions, GRMs require fewer products than SOPs.

Table 4.4 compares the number of products to realize other benchmark functions. ${ }^{2}$ This table shows that, in many cases, GRMs require fewer products than SOPs. For adders and some other functions, GRMIN produced exact minimum GRMs. By solving a recurrence relation generated from the experimental results, we found that the minimum number of products required by GRM for $\operatorname{adr} n$ is $2^{n+1}+n-2$.

\footnotetext{
${ }^{2}$ In this table $\operatorname{adr} n$ is an $n$-bit adder without carry input, inc $n$ increments an $n$-bit binary number, and $\operatorname{wg} \mathbf{t} n$ counts the number of 1-bits in an $n$-bit binary number.
} 
TABLE 4.4

NUMBER OF PRODUCTS TO REALIZE OTHER BENCHMARK FUNCTIONS

\begin{tabular}{|c|c|c|c|c|c|}
\hline Data & In & Out & GRM & ESOP & SOP \\
\hline $5 x p 1$ & 7 & 10 & 35 & 32 & 63 \\
\hline $\operatorname{adr} 4$ & 8 & 5 & 34 * & 31 & 75 \\
\hline $\operatorname{adr} 5$ & 10 & 6 & $67 *$ & 63 & 167 \\
\hline $\operatorname{adr} 6$ & 12 & 7 & 132 * & 127 & 355 \\
\hline $\operatorname{adr} 7$ & 14 & 8 & $261 *$ & 255 & 535 \\
\hline $\operatorname{adr} 8$ & 16 & 9 & $518 *$ & 511 & 1499 \\
\hline $\operatorname{adr} 9$ & 18 & 10 & $1031 *$ & 1023 & 3031 \\
\hline $\operatorname{adr} 10$ & 20 & 11 & 2056 * & 2047 & 6099 \\
\hline al2 & 16 & 47 & 69 & 68 & 66 \\
\hline alu1 & 12 & 8 & 16 & 16 & 19 \\
\hline and & 14 & 24 & 71 & 58 & 66 \\
\hline $\mathrm{b} 12$ & 15 & 9 & 28 & 28 & 44 \\
\hline b9 & 16 & 5 & 81 & 81 & 119 \\
\hline $\operatorname{chkn}$ & 29 & 7 & 151 & 145 & 141 \\
\hline clip & 9 & 5 & 117 & 67 & 117 \\
\hline cps & 24 & 109 & 175 & 137 & 172 \\
\hline duke2 & 22 & 29 & 87 & 81 & 86 \\
\hline gary & 15 & 11 & 116 & 102 & 107 \\
\hline in 2 & 19 & 10 & 122 & 108 & 134 \\
\hline inc 12 & 12 & 13 & $23 *$ & 23 & 79 \\
\hline inc13 & 13 & 14 & $25 *$ & 25 & 92 \\
\hline intb & 15 & 7 & 375 & 327 & 629 \\
\hline life & 9 & 1 & 51 & 49 & 84 \\
\hline $\log 8 \bmod$ & 8 & 5 & 30 & 30 & 38 \\
\hline luc & 8 & 27 & 34 & 28 & 26 \\
\hline $\mathrm{m} 181$ & 15 & 9 & 29 & 29 & 41 \\
\hline $\mathrm{m} 3$ & 8 & 16 & 57 & 51 & 62 \\
\hline
\end{tabular}

\begin{tabular}{|c|c|c|c|c|c|}
\hline Data & In & Out & GRM & ESOP & SOP \\
\hline $\mathrm{m} 4$ & 8 & 16 & 95 & 84 & 101 \\
\hline $\max 1024$ & 10 & 6 & 324 & 165 & 262 \\
\hline $\max 128$ & 7 & 24 & 86 & 63 & 78 \\
\hline $\max 46$ & 9 & 1 & 47 & 41 & 46 \\
\hline $\max 512$ & 9 & 6 & 151 & 84 & 133 \\
\hline $\operatorname{misex} 2$ & 25 & 18 & 27 & 27 & 28 \\
\hline misex 3 & 14 & 14 & 764 & 553 & 696 \\
\hline $\operatorname{mlp} 6$ & 12 & 12 & 1277 & 862 & 1870 \\
\hline newbyte & 5 & 8 & 8 & 8 & 8 \\
\hline newcpla1 & 9 & 16 & 34 & 33 & 38 \\
\hline newxcpla & 9 & 23 & 34 & 30 & 41 \\
\hline radd & 8 & 5 & $34 *$ & 31 & 75 \\
\hline rckl & 32 & 7 & 32 & 32 & 32 \\
\hline $\operatorname{rd} 53$ & 5 & 3 & $20 *$ & 14 & 31 \\
\hline $\operatorname{rd} 73$ & 7 & 3 & $63 *$ & 35 & 127 \\
\hline $\operatorname{rd} 84$ & 8 & 4 & $107^{*}$ & 58 & 255 \\
\hline risc & 8 & 31 & 27 & 26 & 27 \\
\hline sao2 & 10 & 4 & 28 & 28 & 58 \\
\hline sqr6 & 6 & 12 & 35 & 34 & 47 \\
\hline sym10 & 10 & 1 & 110 & 82 & 210 \\
\hline sym9 & 9 & 1 & $126^{*}$ & 51 & 84 \\
\hline t1 & 21 & 23 & 94 & 90 & 103 \\
\hline $\mathrm{t} 481$ & 16 & 1 & 13 & 13 & 481 \\
\hline ts 10 & 22 & 16 & 128 & 128 & 128 \\
\hline wgt10 & 10 & 4 & 310 * & 143 & 1023 \\
\hline wgt12 & 12 & 4 & 1068 * & 445 & 4095 \\
\hline $\mathrm{z} 4$ & 7 & 4 & $32 *$ & 29 & 59 \\
\hline
\end{tabular}

\section{CONCLUSion}

In this paper, we presented GRMIN, a heuristic simplification algorithm for GRMs of multiple-output functions. GRMs have easily testable realizations. Experimental results show that, in most cases, GRMs require fewer products than SOPs. Also, we showed that GRMIN outperforms existing algorithms. For adders and some other functions, GRMIN produced exact minimum solutions.

\section{ACKNOWLEDGEMENT}

This work was supported in part by a Grant in Aid for the Scientific Research of the Ministry of Education, Science and Culture of Japan.

\section{REFERENCES}

[1] D. Brand, and T. Sasao, "Minimization of AND-EXOR expressions using rewrite rules," IEEE Trans. Comput., vol. 42, No. 5, pp. 568-576, May 1993.

[2] M. Cohn, "Inconsistent canonical forms of switching functions," IRE Trans., EC-11, pp. 284-285, Apr. 1962.

[3] L. Csanky, M. A. Perkowski, and I. Schäfer, "Canonical restricted mixed-polarity exclusive-OR sums of products and the efficient algorithm for their minimisation," IEE Proceedings-E, vol. 140, No. 1, pp. 69-77, Jan. 1993.

[4] M. Davio, J-P Deschamps, and A. Thayse, Discrete and Switching Functions, McGraw-Hill Int., New York, 1978.

[5] H. Fujiwara, Logic Testing and Design for Testability, The MIT Press, Cambridge, 1985.

[6] D. Green, Modern Logic Design, Addison-Wesley Publishing Company, Wokingham, England, 1986.
[7] S. J. Hong, R. G. Cain, and D. L. Ostapko, "MINI: A heuristic approach for logic minimization," IBM J. Res. Es Develop. pp. 443-458, Sept. 1974.

[8] A. Mukhopadhyay, and G. Schmitz, "Minimization of ExClusive OR and Logical Equivalence switching circuits," IEEE Trans. Comput., vol. C-19, pp. 132-140, Feb. 1970.

[9] M. A. Perkowski, L. Csanky, A. Sarabi, and I. Schäfer, "Fast minimization of mixed-polarity AND-XOR canonical networks," Proc. International Conference on Computer Design 1992, pp. 33-36, Oct. 1992.

[10] S. M. Reddy, "Easily testable realizations for logic functions," IEEE Trans. Comput., vol. C-21, No. 11, pp. 11831188, Nov. 1972.

[11] T. Sasao, and P. Besslich, "On the complexity of MOD-2 sum PLA's," IEEE Trans. Comput., vol. 39, No. 2, pp. 262-266, Feb. 1990.

[12] T. Sasao, "AND-EXOR expressions and their optimization," in (Sasao e.d.) Logic Synthesis and Optimization, Kluwer Academic Publishers, 1993.

[13] T. Sasao, "EXMIN2: A simplification algorithm for exclusive-OR sum-of-products expressions for multiplevalued input two-valued output functions," IEEE Trans. CAD., vol. 12, No. 5, pp. 621-632, May 1993.

[14] T. Sasao, "Easily testable realizations for generalized Reed-Muller expressions," Proc. IEEE The Third Asian Test Symposium, pp. 157-162, Nov. 1994.

[15] T. Sasao, and D. Debnath, "An exact minimization algorithm for generalized Reed-Muller expressions," Proc. IEEE Asia-Pacific Conference on Circuits $B$ Systems, pp. 460-465, Dec. 1994. 